\title{
Responsividade materna e desenvolvimento infantil de prematuros nascidos em um hospital público e um privado: uma análise comparativa
}

\author{
Maternal responsiveness and child development of preterm infants born in a public and a private \\ hospital: a comparative analysis \\ Capacidad de respuesta materna y desarrollo infantil de los recién nacidos prematuros nacidos en \\ un hospital público y un privado: un análisis comparativo
}

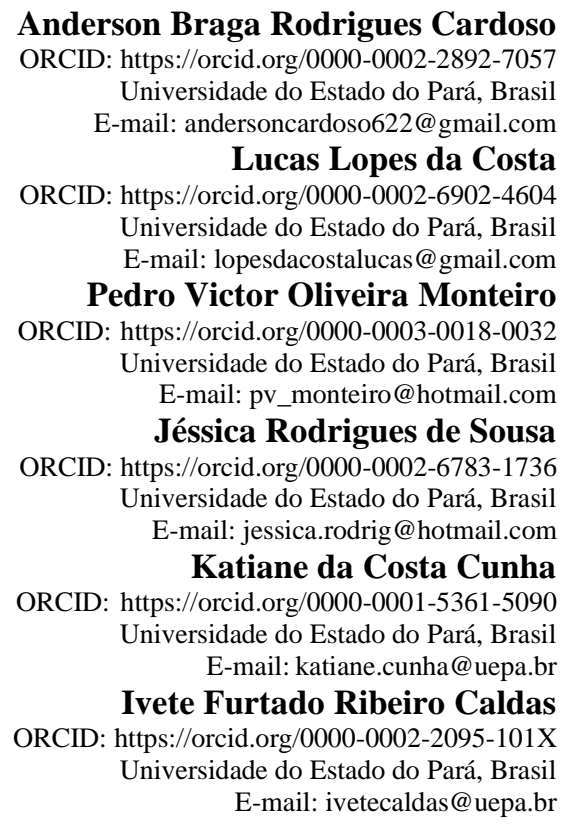

\begin{abstract}
Resumo
O primeiro ano de vida de uma criança é um dos períodos de maior suscetibilidade à influência de fatores protetores ou de risco, os quais podem repercutir até a vida adulta. Dessa forma, a responsividade materna (RM) ou a capacidade da mãe de detectar os sinais comportamentais do bebê e responder a eles, é componente-chave. Nesse sentido, objetivouse analisar comparativamente o comportamento materno e o desfecho do desenvolvimento infantil de prematuros nascidos em um hospital público e um privado durante o primeiro ano de vida. Participaram do estudo 20 díades, sendo 12 do Hospital Privado (HPr) e 8 do Hospital Público (HPu), sendo mães de neonatos com < 36 6/7 semanas e com peso ao nascer $<2.600$ gramas. Foram utilizados a ficha clínica e socioeconômica, o Teste de Desenvolvimento Denver II (DENVER II) e a Escala de Interação Social (EIS). Os dados foram analisados com o Teste G e p-valor de 0.05 . Dentre as mães, $60 \%$ estavam na faixa etária de 30 a 39 anos, das que possuíam ensino superior completo, $12.5 \%$ eram do HPu e 58.4\% do HPr, com relação a renda familiar de 3 a 5 salários mínimos, 37.5\% eram do HPu e $100 \%$ do HPr. O domínio afeto apresentou-se significativamente maior entre as díades do HPr durante todo o período de avaliação na EIS e no desfecho DENVER II. Portanto, evidenciou-se que a interação materna é imprescindível para o comportamento e as habilidades dos prematuros, refletindo-se diretamente no crescimento e desenvolvimento, principalmente no primeiro ano de vida.
\end{abstract}

Palavras-chave: Comportamento materno; Desenvolvimento infantil; Recém-nascido prematuro.

\begin{abstract}
The first year of a child's life is one of the periods of greatest susceptibility to the influence of protective or risk factors, which can affect adulthood. Thus, maternal responsiveness (MR) or the mother's ability to detect the baby's behavioral signs and respond to them, is a key component. In this sense, the objective was to comparatively analyze the maternal behavior and the outcome of child development of preterm infants born in a public and a private hospital during their first year of life. Twenty dyads attended the study, 12 from the Private Hospital (PrH) and 8 from the Public Hospital
\end{abstract}


$(\mathrm{PuH})$, being mothers of newborns $<366 / 7$ weeks and with birth weight $<2.600$ grams. The clinical and socioeconomic form, the Denver II Development Test (DENVER II) and the Social Interaction Scale (SIS) were used. Data were analyzed with the G-Test and p-value of 0.05 . Among the mothers, $60 \%$ were aged between 30 and 39 years, of those who were graduates, $12.5 \%$ were from $\mathrm{PuH}$ and $58.4 \%$ from $\mathrm{PrH}$, in relation to family income of 3 to 5 minimum wages, $37.5 \%$ were from $\mathrm{PuH}$ and $100 \%$ from $\mathrm{PrH}$. The affect domain was significantly higher among the PrH dyads throughout the evaluation period in the SIS and in the DENVER II outcome. Therefore, it was shown that maternal interaction is essential for the behavior and skills of preterm infants, directly reflecting on growth and development, especially in their first year of life.

Keywords: Maternal behavior; Child development; Infant, premature.

\section{Resumen}

El primer año de vida de un niño es uno de los períodos de mayor suscetibilidad a la influencia de factores de protección o de riesgos, los cuales pueden propagarse hasta la vida adulta. Por ello, la responsividad materna (RM) o capacidad de la madre de identificar y responder señales de comportamiento del bebé es um componente clave. En este sentido, el objetivo fue analizar comparativamente el comportamiento materno y el resultado del desarrollo infantil de los prematuros nacidos en un hospital público y un privado durante el primer año de vida. Participaron del estudo 20 díadas, siendo 12 de hospital privado (HPr) y 8 de hospital público ( $\mathrm{HPu}$ ), madres de neonatos con < 36 6/7 semanas y peso < 2.600 gramos al nacer. Fueron utilizados ficha clínica y socioeconômica, el Test de Desarrollo Denver II (DENVER II) y Escala de Interacción Social (EIS). Además, los datos fueron analisados con el Test G y p-valor de 0.05. Entre las madres, el 60\% estaban en el grupo de edad de 30 a 39 años, de las que poseían educación superior completa, el 12.5\% eran de $\mathrm{HPu}$ y el 58.4\% de HPr. Con relación a la renta familiar de 3 a 5 salarios mínimos, el 37.5\% eran de $\mathrm{HPu}$ y el $100 \%$ de HPr. El dominio afecto se presentó significativamente mayor entre las díades del HPr durante todo el período de evaluaciones en la EIS y en el resultado DENVER II. Por consiguiente, se evidenció que la interacción materna es imprescindible para el comportamiento y las habilidades de los prematuros, reflejándose directamente en el crecimiento y desarrollo, principalmente en el primer año de vida.

Palabras clave: Conducta materna; Desarrollo infantil; Recién nacido prematuro.

\section{Introdução}

O desenvolvimento humano é caracterizado pela capacidade contínua do indivíduo em se adaptar ao ambiente em que está inserido. É marcado por profundas interações biopsicossociais que se expressam de forma gradual, à medida que o indivíduo atinge a competência de corresponder às suas necessidades e as do ambiente (Barreto, 2016). Dessa maneira, o desenvolvimento infantil (DI) contempla quatro grandes áreas: "Pessoal-Social", referente à socialização da criança; "Motor Grosso", relativo à administração motora do corpo; "Motor Fino-Adaptativo", que compreende os movimentos de preensão, e a "Linguagem”, a qual engloba o reconhecimento, entendimento e utilização do código linguístico (Costa et al., 2020).

Os primeiros anos de vida da criança são fundamentais para o seu desenvolvimento neuropsicomotor, pois é nesse período que eles estão mais suscetíveis à influência de fatores protetores ou de risco, os quais podem repercutir até a vida adulta. Em vista disso, indivíduos que possuem limitações inerentes ao processo adaptativo, tendem a apresentar dificuldades físicas e comportamentais em curto, médio e/ou longo prazo, com repercussões importantes na qualidade de vida futura (Araújo \& Israel, 2017).

Nesse contexto, outros fatores também podem influenciar o DI, sendo a qualidade da relação precoce mãe-bebê um deles (Feldman et al., 2014; Caldas, Garotti et al., 2018). Variações na qualidade do apego estão associadas a diferenças de personalidade identificadas mais tarde na vida. Um componente-chave dessa relação é a responsividade materna (RM) ou a capacidade da mãe de detectar os sinais comportamentais do bebê e responder a eles. Mães e bebês possuem uma predisposição perceptual e comportamental para se envolver em interações que promovem o apego (Feldman et al., 2014).

Entretanto, as condições clínicas dos bebês, como a prematuridade, caracterizada pelo nascimento antes de 37 semanas de gestação, podem apresentar desafios para as interações entre mães e filhos (Monteiro et al., 2020). As interações maternas com bebês prematuros são menos eficazes que as interações com bebês nascidos a termo. Os longos períodos de hospitalização influenciam diretamente nessa relação, e o desenvolvimento desses prematuros pode tornar-se comprometido em virtude das interações mãe-bebê tão restritas (Mesquita et al., 2020). A presença de ansiedade materna em hospitalizações prolongadas 
resulta em comportamentos maternos menos eficazes durante as interações com seus filhos nos primeiros meses de vida (Feldman et al., 2014).

Felizmente, estratégias de humanização como o contato pele a pele, proporcionado imediatamente após o parto, pode acalmar, auxiliar na estabilização da frequência cardíaca e respiratória, diminuir o choro e estresse, manter o recém-nascido (RN) aquecido pelo calor materno e, principalmente, ajuda na criação de afeto imediato entre a mãe e o bebê. O vínculo criado facilita a amamentação na primeira hora de vida, fortalecendo o contato e interação da díade (Matos et al., 2010; Silva et al., 2018). Esses importantes passos na assistência ao RN, devem ser estimulados pela equipe multiprofissional que o assiste, principalmente em casos de RN's prematuros internados (Brasil, 2012; Nascimento \& Issle, 2004).

Todavia, no Brasil, o conhecimento e boas práticas de assistência médica e hospitalar ainda tem se mostrado insuficiente, desfavorecendo a qualidade da vinculação mãe-bebê. Moreira et al. (2014), em um estudo retrospectivo de abrangência nacional de hospitais públicos e privados, destaca baixas proporções de estímulo ao contato pele a pele (28.3\%) e oferta do seio materno na sala de parto $(16.1 \%)$, com índices razoáveis apenas para a amamentação na primeira hora de vida (47.9\%); tratando-se especificamente da região norte, os valores são ainda menores, exceto para a amamentação na primeira hora $(27.9 \%, 12.5 \%$ e $57.9 \%)$.

Dessa forma, fatores como prematuridade, necessidade de hospitalização frequente, desconhecimento das boas práticas assistenciais ao RN, ineficiência na formação de vínculos da díade e o desconhecimento materno da importância de um ambiente de estímulos, podem influenciar no processo no desenvolvimento de habilidades infantis. Nesse sentido, visando contribuir para melhor compreensão do impacto do comportamento materno no DI, o objetivo deste estudo consistiu em analisar o comportamento materno e o desfecho do desenvolvimento infantil de prematuros nascidos em um hospital público e um privado durante o primeiro ano de vida.

\section{Metodologia}

Trata-se de um estudo de coorte prospectivo, comparativo, com amostra por conveniência. Esse tipo de estudo também é chamado de estudo longitudinal ou de follow-up, partindo-se da premissa de que o pesquisador irá acompanhar uma determinada população por um período de tempo em busca de possíveis achados relacionados à exposição a determinados fatores e os possíveis desfechos encontrados (Camargo et al., 2019).

Um total de 33 mães com seus neonatos foram recrutados. Sendo 13 díades de um hospital privado, localizado no município de Belém, Pará, em que foi excluída uma díade por óbito neonatal. E 20 díades de um hospital público, localizado no município de Marabá, Pará, em que foram excluídas 10 por dificuldade de contato e duas por desistência. Atribuiu-se a essa perda expressiva da amostra do hospital público, o fato de as mães residirem em zonas rurais, onde o transporte público é ineficiente e o acesso ao sinal de telefonia é precário, além da ausência de um serviço de follow-up multidisciplinar no município.

Assim, participaram do estudo 12 díades do hospital privado (HPr) e 8 do público (HPu). Em ambos os locais eram mães com seus respectivos neonatos com idade gestacional (IG) < 36 6/7 semanas e peso ao nascer (PN) < 2.600 gramas. Foram excluídas mães com diagnósticos psiquiátricos, recém-nascidos com malformações congênitas e síndromes genéticas detectadas no período neonatal. Este estudo foi aprovado pelo Comitê de Ética em Pesquisa (Parecer $n^{0}$ 176.898).

Para coleta de dados, utilizaram-se os seguintes instrumentos:

Ficha clínica e socioeconômica: Fornece dados de identificação da mãe, do cônjuge e da criança (endereço, idade, etc.), dados maternos e gestacionais (doenças obstétricas, complicações no parto, número de gestações, tipo de parto, pré-natal, etc.), neonatais (peso ao nascer, idade gestacional, apgar, sexo, etc.) e pós-natais (necessidade de suporte ventilatório, drogas vasoativas, fototerapia, presença de complicações neonatais, etc.) que foram coletados dos prontuários dos respectivos recém- 
nascidos prematuros.

Teste de Triagem do Desenvolvimento de Denver II (TTDD II): Representa um teste de triagem não diagnóstico. Tem como objetivo avaliar o desenvolvimento das crianças do nascimento aos 6 anos de idade, é composto por 125 itens distribuídos nas seguintes categorias: pessoal-social, motor fino-adaptativo, linguagem e motor grosso. Em cada uma das habilidades avaliadas foram consideradas três possibilidades de pontuações dos itens que são: passa $(\mathrm{P})$, falha $(\mathrm{F})$, recusa $(\mathrm{R})$ ou sem oportunidade (SO). Após a avaliação, as crianças foram classificadas conforme seu desempenho: "normal", quando não houve nenhum atraso ou no máximo um cuidado/cautela em pelo menos uma área; "risco", para duas ou mais cautelas e/ou um atraso em pelo menos uma área; e "atraso", quando se obtiveram dois ou mais itens de atraso, apontando que a criança apresenta grande suspeita de alteração do desenvolvimento (Frankenburg et al., 1992).

Escala de Interação Social (EIS): Verifica a responsividade do cuidador quanto ao nível de afeto, manutenção da interação, diretividade/intrusividade, contingência, envolvimento e participação nas interações. Cada dimensão foi pontuada de acordo com uma escala do tipo Likert de 5 pontos (1, nível quantitativo menor; $1.5 ; 2 ; 2.5 ; 3)$. De acordo com a pontuação final, que corresponde a soma das pontuações atribuídas a cada dimensão/domínio, as interações foram classificadas com "pobre” (610 pontos), "boa" (10.5-14.5 pontos) ou “ótima" (15-18 pontos) (Ruble et al., 2008).

Em ambos os grupos, depois dos esclarecimentos sobre o objetivo da pesquisa e após a assinatura do Termo de Consentimento Livre e Esclarecido (TCLE), estabeleceu-se um cronograma de sessões ao longo do primeiro ano de vida. Trimestralmente, as sessões ocorreram, equivalendo aos três, seis, nove e doze meses de idade (idade cronológica). Porém, em virtude de a amostra ser composta por prematuros, fez-se necessário o cálculo da idade corrigida (ICg), que visou adequar o desenvolvimento das crianças a cada idade registrada. Assim, as ICgs corresponderam em média, respectivamente, a um, quatro, sete e dez meses.

As sessões foram desenvolvidas de forma semiestruturada, no horário diurno, e registradas em vídeo por alunos de iniciação científica treinados. No grupo HPr as sessões foram realizadas em um consultório do mesmo hospital privado em que os prematuros nasceram, localizado na capital paraense, e no grupo HPu, no Laboratório de Desenvolvimento Infantil (LADIN) do Campus VIII da Universidade do Estado do Pará (UEPA), no município de Marabá, Pará. Durante a consulta de follow-up do prematuro, dependendo da idade da criança, foram selecionados diferentes brinquedos.

As sessões tiveram em média duração de (30) trinta minutos. Nos (10) dez primeiros minutos foi realizada a coleta das informações clínicas da mãe e do prematuro para preencher a ficha clínica e socioeconômica. Nos (10) dez minutos seguintes foram realizadas avaliação do desenvolvimento infantil dos recém-nascidos através do Denver II. Nos (10) dez minutos finais as mães foram orientadas a interagirem livremente com seus filhos (interação mãe-criança). Nesta pesquisa, foram analisados os seis minutos finais do vídeo referente ao período de interação livre mãe-bebê, com o intuito de avaliar a responsividade materna (RM). Dois avaliadores treinados analisaram os vídeos para o cálculo do índice de concordância referente aos comportamentos da mãe e da criança.

Os dados provenientes dos instrumentos foram tabulados no programa Microsoft Excel® e analisados no software Statistical Package for Social Sciences (SPSS) versão 22.0. Foram realizadas análises comparativas, estatísticas descritivas e inferenciais quanto aos resultados dos grupos pesquisados. A variável dependente da pesquisa foi o escore de desenvolvimento advindo do Denver II, tratada como variável dicotômica (normal, risco ou atraso). As variáveis independentes foram obtidas da EIS.

Visando analisar a associação desfecho entre as variáveis ao final das quatros sessões, foi utilizado o Teste G, adotandose o nível de significância de 5\% (p valor <0.05). O índice de concordância entre os observadores independentes referente aos dois grupos, previamente treinados, foi calculado com base em 35\% do total das sessões. No grupo HPr o índice de concordância foi de $83 \%$ tanto para os resultados do escore da RM quanto para o Denver II, enquanto no grupo $\mathrm{HPu}$, foi de $92 \%$ para a RM e 
$88 \%$ para o Denver II.

\section{Resultados}

A Tabela 1 detalha as características socioeconômicas e os aspectos clínicos das díades mãe-bebê relacionado aos grupos HPr e HPu. Em relação a faixa etária materna de 30-39 anos, 4 (50.0\%) mães pertencem ao grupo HPu e 8 (66.7\%) mães ao HPr. No tocante as mães que tinham ensino superior completo, apenas 1 (12.5\%) mãe pertencia ao grupo HPu, enquanto 7 (58.4\%) ao HPr. No que se refere a renda familiar de 3 a 5 salários mínimos, 3 (37.5\%) mães faziam parte do grupo HPu e 12 $(100 \%)$ mães do HPr.

Além disso, em referência a idade gestacional de 32 a 37 semanas e peso ao nascer entre 1500 a $2500 \mathrm{~g}, 6(75.0 \%)$ crianças nasceram no HPu e 10 (83.4\%) no HPr. Do total de crianças avaliadas observou-se uma proporção de 50\% entre os sexos. Acerca do apgar no $5^{\circ}$ minuto, 8 (100\%) crianças do grupo HPu obtiveram valor acima de 7, por sua vez, apenas 1 (8.4\%) criança do HPr obteve valor inferior a 7. Daquelas crianças que necessitaram de suporte ventilatório por mais que três dias, 3 (37.5\%) pertenciam ao grupo HPu e 8 (66.7\%) ao HPr, e que apresentaram icterícia neonatal, 3 (37.5\%) eram do HPu e 5 (41.7\%) do HPr. 
Tabela 1. Características socioeconômicas das famílias e aspectos clínicos mãe-bebê relacionado aos grupos Hospital Público (HPu) e Hospital Privado (HPr).

\begin{tabular}{|c|c|c|c|}
\hline \multirow{2}{*}{ Variáveis } & \multirow{2}{*}{$\begin{array}{c}\text { Amostra } \\
\mathbf{N}=\mathbf{2 0} \\
\text { Total } \\
\mathbf{N}(\%)\end{array}$} & \multicolumn{2}{|c|}{ Grupo } \\
\hline & & $\begin{array}{c}\text { HPu } \\
\text { n (\%) }\end{array}$ & $\begin{array}{c}\mathrm{HPr} \\
\mathrm{n}(\%)\end{array}$ \\
\hline \multicolumn{4}{|l|}{ Idade materna (anos) } \\
\hline Menor que 18 & $1(5.0)$ & $1(12.5)$ & $0(0.0)$ \\
\hline $18-29$ & $4(20.0)$ & $2(25.0)$ & $2(16.6)$ \\
\hline $30-39$ & $12(60.0)$ & $4(50.0)$ & $8(66.7)$ \\
\hline $40-49$ & $3(15.0)$ & $1(12.5)$ & $2(16.6)$ \\
\hline \multicolumn{4}{|l|}{ Educação materna } \\
\hline Ensino Médio Incompleto & $3(15.0)$ & $2(25.0)$ & $1(8.3)$ \\
\hline Ensino Médio Completo & $6(30.0)$ & $2(25.0)$ & $4(33.3)$ \\
\hline Ensino Superior Incompleto & $3(15.0)$ & $3(37.5)$ & $0(0.0)$ \\
\hline Ensino Superior Completo & $8(40.0)$ & $1(12.5)$ & $7(58.4)$ \\
\hline \multicolumn{4}{|l|}{ Renda familiar } \\
\hline Até 1 Salário Mínimo & $1(5.0)$ & $1(12.5)$ & $0(0.0)$ \\
\hline Entre 1 e 3 Salários Mínimos & $4(20.0)$ & $4(50.0)$ & $0(0.0)$ \\
\hline Entre 3 e 5 Salários Mínimos & $15(75.0)$ & $3(37.5)$ & $12(100)$ \\
\hline \multicolumn{4}{|l|}{ Idade gestacional } \\
\hline 28 a 31 semanas & $4(20.0)$ & $2(25.0)$ & $2(16.6)$ \\
\hline 32 a 37 semanas & $16(80.0)$ & $6(75.0)$ & $10(83.4)$ \\
\hline \multicolumn{4}{|l|}{ Peso ao nascer } \\
\hline $1000 \mathrm{a}<1500 \mathrm{~g}$ & $3(15.0)$ & $1(12.5)$ & $2(16.6)$ \\
\hline $1500 \mathrm{a} \leq 2500 \mathrm{~g}$ & $16(80.0)$ & $6(75.0)$ & $10(83.4)$ \\
\hline$<2600$ & $1(5.0)$ & $1(12.5)$ & $0(0.0)$ \\
\hline \multicolumn{4}{|l|}{ Sexo } \\
\hline Masculino & $10(50.0)$ & $5(62.5)$ & $5(41.6)$ \\
\hline Feminino & $10(50.0)$ & $3(37.5)$ & $7(58.4)$ \\
\hline \multicolumn{4}{|l|}{ Apgar 5} \\
\hline Até 7 pontos & $1(5.0)$ & $0(0.0)$ & $1(8.4)$ \\
\hline Maior que 7 pontos & $19(95.0)$ & $8(100.0)$ & $11(91.6)$ \\
\hline \multicolumn{4}{|l|}{ Suporte ventilatório } \\
\hline Menos que 3 dias & $9(45.0)$ & $5(62.5)$ & $4(33.3)$ \\
\hline Maior ou igual a 3 dias & $11(55.0)$ & $3(37.5)$ & $8(66.7)$ \\
\hline \multicolumn{4}{|l|}{ Icterícia neonatal } \\
\hline Sim & $12(60.0)$ & $5(62.5)$ & $7(58.3)$ \\
\hline Não & $8(40.0)$ & $3(37.5)$ & $5(41.7)$ \\
\hline
\end{tabular}

Fonte: Dados da pesquisa.

A Tabela 2 apresenta os resultados da Responsividade Materna (RM) para os domínios da Escala de Interação Social (EIS) nos quatro meses estudados. Com relação ao domínio do afeto, observou-se resultados menores que o nível de significância ( $p$ valor $=0.007)$, indicando que houve diferença estatística entre as médias das díades do grupo HPu em relação ao HPr durante os quatro meses estudados. Para os domínios diretividade, iniciação, manutenção, contingência e participação não houve 
significância, indicando que não existiu diferença estatística entre as médias das díades do grupo HPu em relação ao grupo HPr nos meses estudados.

Tabela 2. Resultados da RM para os domínios da EIS nos quatro meses estudados.

\begin{tabular}{|c|c|c|c|c|c|}
\hline Domínios & $\begin{array}{l}\text { Graus de } \\
\text { liberdade }\end{array}$ & $\begin{array}{c}\text { Soma de } \\
\text { quadrados }\end{array}$ & Quadrado médio & Valor F & P-valor \\
\hline Afeto & 1 & 4.21875 & 4.21875 & 7.444 & $0.007 * *$ \\
\hline Resíduos & 78 & 44.20321 & 0.56671 & & \\
\hline Diretividade & 1 & 0.43802 & 0.43802 & 0.7113 & 0.401 \\
\hline Resíduos & 78 & 48.03385 & 0.61582 & & \\
\hline Iniciação & 1 & 0.83333 & 0.83333 & 1.322 & 0.253 \\
\hline Resíduos & 78 & 49.16667 & 0.63034 & & \\
\hline Manutenção & 1 & 0.32552 & 0.32552 & 0.4962 & 0.483 \\
\hline Resíduos & 78 & 51.17448 & 0.65608 & & \\
\hline Contingência & 1 & 0.63802 & 0.63802 & 0.9749 & 0.326 \\
\hline Resíduos & 78 & 51.04948 & 0.65448 & & \\
\hline Participação & 1 & 1.10208 & 1.10208 & 1.782 & 0.185 \\
\hline Resíduos & 78 & 48.24479 & 0.61852 & & \\
\hline
\end{tabular}

Legenda: Teste $\mathrm{G} ; * p<0.05 ; * * p<0.01$. Fonte: Dados da pesquisa.

A Figura 1 evidencia as médias das pontuações da RM para os domínios da EIS nos quatro meses de idade corrigida (IC) estudados para os grupos HPu e HPr. Referente ao domínio afeto, pode-se observar que o grupo HPu se manteve com as médias inferiores ao grupo HPr durante todo o período das avaliações, por sua vez, com relação aos domínios diretividade, iniciação, manutenção, contingência e participação, o grupo HPu obteve uma melhor pontuação média na avalição de um mês de ICg, porém nas avaliações posteriores o grupo HPr obteve os melhores resultados.

Figura 1. Médias das pontuações na RM para os domínios da EIS nos quatro meses IC estudados relacionado aos grupos Hospital Público e Hospital Privado.

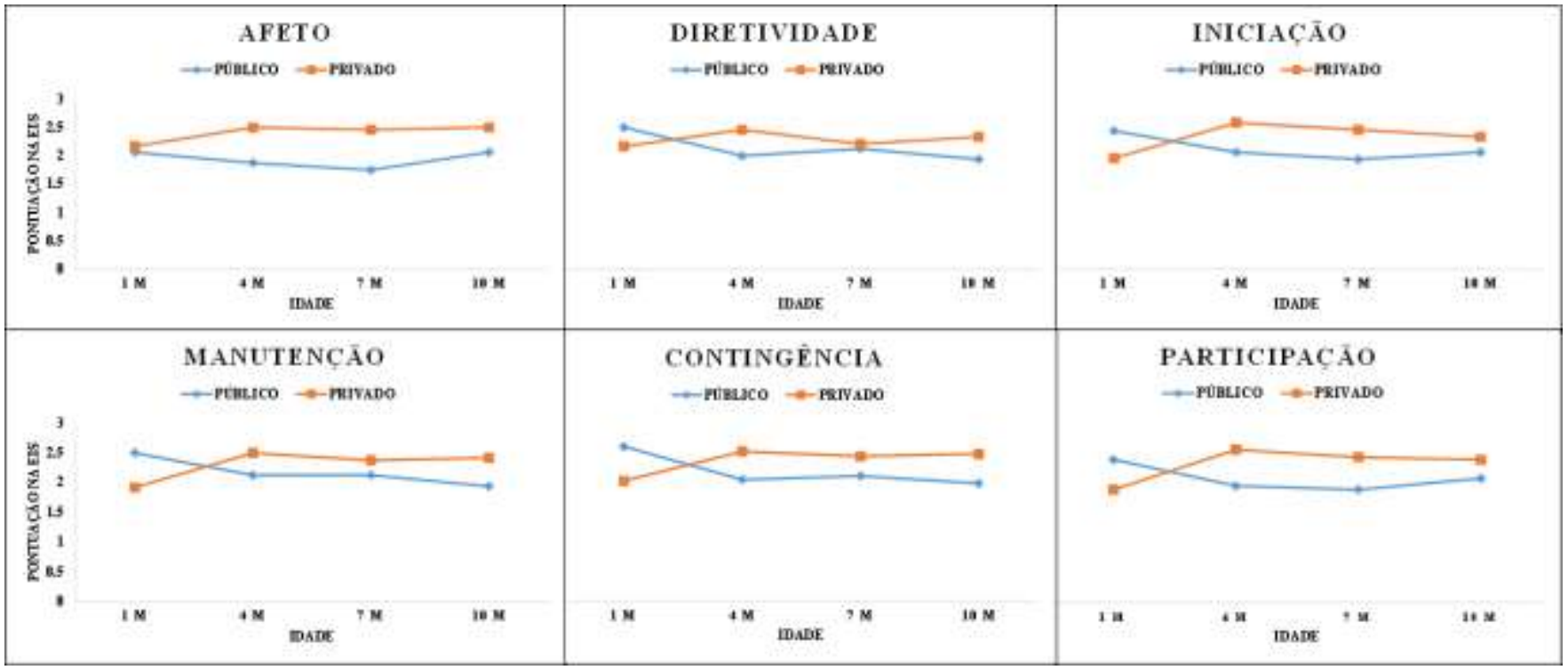

Fonte: Dados da pesquisa.

Por fim, a Tabela 3 apresenta os resultados para os domínios da EIS em relação ao desfecho DENVER II nos quatro meses estudados. Com relação ao domínio do afeto, observou-se resultados menores que o nível de significância $(p$ valor $=$ 
0.006), indicando que houve diferença estatística para o desfecho do Denver II entre as médias das díades do grupo HPu em relação ao HPr durante os quatro meses estudados. Porém, para os domínios diretividade, iniciação, manutenção, contingência e participação não houve significância, indicando que não existiu diferença estatística entre as médias das díades do HPu em relação ao HPr nos meses estudados.

Tabela 3. Resultados para os domínios da EIS em relação ao desfecho DENVER II nos quatro meses estudados.

\begin{tabular}{|c|c|c|c|c|c|}
\hline Anova & $\begin{array}{c}\text { Graus de } \\
\text { liberdade }\end{array}$ & $\begin{array}{c}\text { Soma de } \\
\text { quadrados }\end{array}$ & Quadrado médio & Valor F & P-valor \\
\hline Afeto & 2 & 6.39437 & 3.19719 & 5.278 & $0.006 * *$ \\
\hline Resíduos & 97 & 58.75321 & 0.60570 & & \\
\hline Diretividade & 2 & 2.76365 & 1.38183 & 2.142 & 0.123 \\
\hline Resíduos & 97 & 62.58385 & 0.64519 & & \\
\hline Iniciação & 2 & 3.39333 & 1.69667 & 2.583 & 0.080 \\
\hline Resíduos & 97 & 63.71667 & 0.65687 & & \\
\hline Manutenção & 2 & 2.88552 & 1.44277 & 2.129 & 0.124 \\
\hline Resíduos & 97 & 65.72448 & 0.64664 & & \\
\hline Contingência & 2 & 4.06052 & 2.03056 & 3.002 & 0.054 \\
\hline Resíduos & 97 & 65.59948 & 0.67628 & & \\
\hline Participação & 2 & 3.13271 & 1.56636 & 2.42 & 0.094 \\
\hline Resíduos & 97 & 62.79479 & 0.64737 & & \\
\hline
\end{tabular}

Legenda: Teste $\mathrm{G} ; * p<0.05 ; * * p<0.01$. Fonte: Dados da pesquisa.

\section{Discussão}

A notória inserção da mulher no concorrido mercado de trabalho, a necessidade de uma formação profissional mais qualificada e, portanto, mais demorada, além do avanço dos métodos anticonceptivos, tornou a gestação tardia uma realidade (Aldrighi et al.,2018). Todavia, a idade materna elevada, de forma direta ou independente, está atrelada a maiores índices de prematuridade. Estudos associam o aumento de doenças crônicas, hipertensão gestacional, obesidade materna e o avanço das técnicas de reprodução assistida, ao nascimento pré-termo (Oliveira et al., 2016; Teixeira, et al., 2018). Ademais, o baixo peso ao nascer também é apontado como fator de risco em mulheres acima dos 35 anos de idade (Gravena et al., 2013).

Quando acontece a formação de vínculo e afeto entre a díade mãe-bebê, habitualmente desde a gestação (vinculação pré-natal), há maior probabilidade de contato e estimulações por parte da genitora, assim como receptividade e reatividade do RN. O apego formado nos primeiros contatos físicos, como na amamentação, banho, trocas de fraldas e roupas do RN até o fim do terceiro mês, são decisivos para a construção do vínculo da díade (primeiro período de formação do apego) e nos períodos subsequentes, influenciando consideravelmente o fortalecimento do vínculo formado e de estimulações durante o crescimento da criança (Silva \& Braga, 2019). Nesse sentido, a contemporânea necessidade em dispor de estabilidade financeira para prover um ambiente minimamente adequado aos filhos, tende a favorecer o apego da díade, visto que a mãe tende a dispor mais tempo e atenção ao cuidado da criança (Bernadi et al., 2018).

Um aspecto importante para o desenvolvimento infantil é o desenvolvimento afetivo, vínculo básico caracterizado no apego (Brasil, 2013). O termo vínculo é muito utilizado para referir-se ao estabelecimento e desenvolvimento da interação mãebebê durante a construção desse processo de afetividade, dessa forma, a mãe espera que seu filho afague seu desejo de ser mãe (Barroso et al., 2015). Desse modo, as vantagens da interação precoce mãe-filho são amplamente reconhecidas, contribuindo para o desenvolvimento, sobretudo no âmbito emocional, social e cognitivo da criança e no atendimento de suas necessidades. Segundo estudos, a reciprocidade e a sensibilidade na interação requerem sintonia e articulam-se aos comportamentos dos sujeitos entre si. Trata-se de um processo de engajamento que se torna progressivamente mais complexo à medida que o binômio 
mãe-bebê avança no conhecimento um do outro e aperfeiçoa sua capacidade de ajuste e regulação mútua dos comportamentos (Joaquim et al., 2018).

Desse modo, a prematuridade da criança e suas dificuldades específicas, à separação precoce, à problemática de estimulação e à experiência emocional materna, que levam a comportamentos intrusivos e controladores da mãe, são particularidades que acabam influenciando no processo do vínculo mãe-bebê. Esses comportamentos estão relacionados com as interações limitadas em razão de separação física, falta de oportunidades plenas de contatos com o filho e acolhimento profissional incipiente, ansiedade, culpa, inseguranças e dificuldades para assumir o cuidado (Frello \& Carraro, 2012; Joaquim et al., 2018).

No que se refere a responsividade materna (RM), evidenciou-se que as mães do grupo do hospital privado (HPr) apresentaram melhores médias com relação ao domínio afeto do que o grupo do hospital público (HPu). Isso pode estar relacionado ao fato das mães do grupo HPr terem se preparado melhor para gestar, tanto no nível escolar em que 58.4\% das mães possuía o ensino superior completo, como pelo nível socioeconômico, em que 100\% das mães possuía renda familiar entre 3 e 5 salários mínimos. Segundo um estudo pediátrico realizado nos hospitais de Pelotas-RS, a pouca escolaridade dos pais somado a baixa renda familiar representaram características socioeconômicas importantes para determinação do maior risco de prematuridade, prejuízo no desenvolvimento infantil e menor interação mãe-bebê. Esse estudo explica ainda que, crianças provenientes de famílias mais abastadas receberam maior estimulação por parte dos pais e variadas oportunidades de interação com o meio e com objetos no primeiro ano de vida (Halpern et al., 2000). Ademais, outro estudo evidenciou que o baixo nível de escolaridade dificulta a assistência, uma vez que as mães têm pouca adesão às consultas pré-natais, puerperais e de puericultura, por não saberem da importância e os benefícios dessa assistência (Carvalho et al., 2018).

Além disso, outra condição que pode ter influenciado na diferença dos resultados com relação ao domínio afeto entre os grupos HPu e HPr é a idade materna, já que 83.3\% das mães do grupo HPr tinham pelo menos 30 anos de idade e não havia mães adolescentes nesse grupo. O que segundo Zanettini et al. (2019), mães jovens, adolescentes e/ou primíparas sentem-se despreparadas para desempenhar a maternidade, pela pouca idade e experiência. Além de que, a prematuridade traz consigo suas dificuldades específicas que podem gerar ansiedade, insegurança e culpa nessas mães (Joaquim et al., 2018).

Por outro lado, observou-se que o grupo HPu obteve resultados bem próximos as médias do grupo HPr na avaliação de primeiro mês de idade corrigida no domínio afeto e resultados melhores que o grupo HPr nesse mesmo período nos domínios de diretividade, iniciação, manutenção, contingência e participação. Essas melhores médias do grupo HPu na primeira avaliação da EIS, podem ser referentes ao fato de muitas mães nos três primeiros meses de vida do RN contarem com maior apoio familiar para gerenciar suas demandas, através da divisão de tarefas domiciliares e do cuidado neonatal com familiares, amigos e, principalmente, com as avós. Esse apoio contribui para facilitar a maternidade, promovendo o desenvolvimento de um apego seguro entre a díade mãe-bebê, já que a mãe conta com auxílio, possibilitando-a em estar mais disponível física e emocionalmente para atender de forma adequada as demandas do bebê (Rapoport \& Piccinini, 2011). Por sua vez, esse apoio familiar pode ter sido deficiente para as mães do HPr, que muitas vezes saíram de suas cidades natais e passaram a morar na capital, longe do apoio familiar, para estudar e buscar melhores condições de trabalho. O que segundo estudos, com o capitalismo, a globalização e as inovações tecnológicas, as mulheres estão buscando cada vez mais se especializar e profissionalizar, ocupando cargos de liderança em grandes empresas (Baylão \& Schettino, 2014). Além disso, muitas mulheres também ficam longe do apoio familiar por terem que acompanhar seus cônjuges que recebem proposta de emprego ou passam em concurso públicos em outras cidades.

No que se refere ao desenvolvimento infantil (DI), compreender e detectar precocemente as alterações é uma tarefa complexa que requer avaliações sistemáticas, especialmente durante o primeiro ano de vida. Nesse período, o crescimento e desenvolvimento é mais dinâmico, há mais capacidade de adaptação, uma maior plasticidade neural, portanto, quanto mais cedo os problemas forem detectados, mais precoce poderá haver uma abordagem multidisciplinar, envolvendo profissionais de várias 
áreas de atuação, tanto da saúde como da educação para auxiliar no DI de forma adequada (Halpern et al., 2000; Eickmann et al., 2012).

Quanto a relação entre os domínios da EIS e o Desfecho DENVER II, evidenciou-se que houve diferença estatística no domínio afeto entre o grupo HPr e grupo HPu, indicando que os melhores resultados no domínio afeto da EIS referente ao grupo HPr refletiram-se no desenvolvimento geral das crianças, evidenciado através do desfecho do Denver II ao final do primeiro ano de vida. Esse DI está diretamente relacionado a estimulação mãe-bebê, além de outros fatores como a interação paterna, familiar, tempo e qualidade das interações, o que favorecem o desenvolvimento global das crianças (Ribeiro et al., 2017).

\section{Considerações Finais}

Pode-se evidenciar através desta pesquisa a importância da interação do binômio mãe-bebê para o desenvolvimento infantil, ainda mais quando se trata de recém-nascidos prematuros. Observou-se as que mães que pertenciam ao grupo do hospital privado tinham maior nível escolar e melhores condições socioeconômicas, além de não possuir nenhuma integrante adolescente nesse grupo. Todos esses fatores, direta ou indiretamente influenciaram em uma melhor responsividade materna, principalmente em relação ao domínio afeto da Escala de Interação Social e no desenvolvimento infantil, evidenciado no desfecho DENVER II ao final do primeiro ano de vida das crianças. Percebeu-se também, que os melhores resultados do grupo do hospital público foram encontrados no primeiro mês de idade corrigida ou terceiro mês de idade cronológica em todos os domínios, tendo melhores resultados que o grupo privado, com exceção apenas para o domínio afeto, evidenciando que essas mães do grupo público podem ter tido um melhor suporte familiar nesse período, o que as auxiliou nas suas demandas e propiciou melhor interação entre a díade mãe-bebê.

Dessa forma, entende-se que esta pesquisa traz uma contribuição para literatura quanto a compreensão da importância da responsividade materna para o desenvolvimento infantil de prematuros acompanhados durante o primeiro ano de vida, pois colabora para a compreensão das interações em um contexto específico. Porém, nessa pesquisa, não participaram recém-nascidos a termo (grupo controle), o que é uma limitação do estudo. Portanto, reiteramos a importância de pesquisas com amostras maiores, contextos culturais diversificados e outras diferenças nas interações, buscando validar ainda mais os resultados encontrados e incentivar uma melhor compreensão da relação mãe e filho, e dessa forma, buscar melhorias na assistência prénatal e neonatal.

\section{Agradecimentos}

Agradecemos ao Conselho Nacional de Desenvolvimento Científico e Tecnológico (CNPq) e a Fundação Amazônia de Amparo à Estudos e Pesquisa (FAPESPA) pela oportunidade de participarmos do Programa Institucional de Bolsas de Iniciação Científica (PIBIC).

\section{Referências}

Aldrighi, J. D., Wall, M. L., \& Souza, S. R. R. K. (2018). Vivências de mulheres na gestação em idade tardia. Revista Gaúcha de Enfermagem, 39, 1-9. https://doi.org/10.1590/1983-1447.2018.2017-0112.

Araújo, L. B., \& Israel V. L. (2017). Desenvolvimento da criança: família, escola e saúde. Curitiba: Omnipax. https://doi.org/10.7436/2017.dcfes.0.

Barreto, A. C. (2016). Paradigma sistêmico no desenvolvimento humano e familiar: a teoria bioecológica de Urie Bronfenbrenner. Psicologia em Revista, 22(2), 275-293. https://doi.org/DOI-10.5752/P.1678-9523.2016V22N2P275.

Barroso, M. L., Pontes, A. L., \& Rolim, K. M. C. (2015). Consequências da prematuridade no estabelecimento do vínculo afetivo entre mãe adolescente e recémnascido. Revista RENE, 16(2), 168-75. https://doi.org/10.15253/2175-6783.2015000200005.

Baylão, A. 1. S., Schettino, E. M. O. (2014). A Inserção da Mulher no Mercado de Trabalho Brasileiro. XI Simpósio de Excelência em Gestão e Tecnologia. Tema: Gestão do conhecimento para Sociedade. 
Bernardi, D., Féres-Carneiro, T., \& Magalhães, A. S. (2018). Entre o desejo e a decisão: a escolha por ter filhos na atualidade. Contextos Clínicos, 11(2), 161173. https://doi.org/10.4013/ctc.2018.112.02.

Brasil (2012). Ministério da Saúde. Cadernos de Atenção Básica. Saúde da criança: crescimento e desenvolvimento. Brasília.

Brasil (2013). Ministério da Saúde. Secretaria de Atenção à Saúde. Além da sobrevivência: práticas integradas de atenção ao parto, benéficas para a nutrição e a saúde de mães e crianças. Brasília.

Caldas, I. F., Garotti, M. F., Shiramizu, V. K., \& Pereira, A. (2018). The socio-communicativedevelopment of preterm infants is resistant to the negative effects of parity on maternal responsiveness. Frontiers in Psychology, 9(43), 1-8. https://doi.org/10.3389/fpsyg.2018.00043.

Camargo, L. M. A., Silva, R. P. M., Meneguetti, D. O. (2019). Tópicos de metodologia de pesquisa: Estudos de coorte ou cohorte prospectivo e retrospectivo. $J$ Hum Growth Dev, 29(3):433-436. https://doi.org/10.7322/jhgd.v29.9543

Carvalho, J. B. L., Teixeira, G. A., Morais, P. C., Sena, A. V., \& Alves, T. R. M. (2018). Condições socioeconômicas da gestação de bebês prematuros. Revista de Enfermagem UFPE on line, 12(2), 386-90. https://doi.org/10.5205/1981-8963-v12i2a15294p386-390-2018.

Costa, L. L., Marinho, A. M. D., Silva, L. C. C., Cunha, K. C., \& Caldas, I. F. R (2020). Associações entre afetividade dos cuidadores e o desenvolvimento infantil de crianças em situação de acolhimento. Revista Brasileira de Educação e Saúde, 10(3), 136-145. https://doi.org/10.18378/rebes.v10i3.7945.

Eickmann, S. H., Malkes, N. F. A., \& Lima, M. C. (2012). Psychomotor development of preterm infants aged 6 to 12 months. São Paulo Medical Journal, 130(5), 299-306. https://doi.org/10.1590/S1516-31802012000500006.

Feldman, R., Rosenthal, Z., \& Eidelman, A. I. (2014). Maternal-preterm skin-to-skin contact enhances child physiologic organization and cognitive control across the first 10 years of life. Biological Psychiatry, 75(1), 56-64. https://doi.org/10.1016/j.biopsych.2013.08.012.

Frankenburg, W. K., Dodds, J., Archer, P., Shapiro, H., \& Bresnick, B. (1992). The Denver II: a major revision and restandardization of the Denver Developmental Screening Test. Pediatrics, 89(1), 91-7.

Frello, A. T., \& Carraro, T. E. (2012). Enfermagem e a relação com as mães de neonatos em Unidade de Terapia Intensiva Neonatal. Revista Brasileira de Enfermagem, 65(3), 514-521. https://doi.org/10.1590/S0034-71672012000300018.

Gravena, A. A. F., Paula, M. G., Marcon, S. S., Carvalho, M. D. B., \& Pelloso, S. M. (2013). Idade materna e fatores associados a resultados perinatais. Acta Paulista de Enfermagem, 26(2), 130-5. https://doi.org/10.1590/S0103-21002013000200005.

Halpern, R., Elsa, R. J. G., Victora, C. G., Barros, F. C., \& Horta, B. L (2000). Fatores de risco para suspeita de atraso no desenvolvimento neuropsicomotor aos 12 meses de vida. Jornal de Pediatria, 76(6), 421-8.

Joaquim, R. H. V. T., Wernet, M., Leite, A. M., Fonseca, L. M. M., \& Mello, D. F. (2018). Interações entre mães e bebês prematuros: enfoque nas necessidades essenciais. Cadernos Brasileiros de Terapia Ocupacional, 26(3), 580-9. https://doi.org/10.4322/2526-8910.ctoAO1051.

Matos, T. A., Souza, M. S., Santos, E. K. A., Velho, M. B., Seibert, E. R. C., \& Martins, N. M. (2010). Contato precoce pele a pele entre mãe e filho: significado para mães e contribuições para a enfermagem. Revista Brasileira de Enfermagem, 63(6), 998-1004. https://doi.org/10.1590/S0034-71672010000600020.

Mesquita, P. C. S., Siqueira, D. D., Garotti, M. F., \& Caldas, I. F. (2020). Associação entre responsividade materna em função da prole e desenvolvimento motor. Psicologia: Teoria e Prática, 22(1), 127-143. https://doi.org/10.5935/1980-6906/psicologia.v22n1p144-160.

Monteiro, P. V. O., Cardoso, A. B. R., Costa, L. L., Caldas, I. F. R., Cunha, K. C., \& Chermont, A. G. (2020). Associações entre desenvolvimento motor e sociocomunicativo de prematuros e interação mãe-bebê. Revista Brasileira de Educação e Saúde, 10(3), 177-183. https://doi.org/10.18378/rebes.v10i3.7976.

Moreira, M. E. L., Gama, S. G. N., Pereira, A. P. E., Silva, A. A. M., Lansky, S., Pinheiro, R. S., Gonçalves, A. C., \& Leal, M. C. (2014). Práticas de atenção hospitalar ao recém-nascido saudável no Brasil. Caderno de Saúde Pública, 30, 128-139. https://doi.org/10.1590/0102-311X00145213.

Nascimento, M. B. R., \& Issler, H. (2004). Aleitamento materno em prematuros: manejo clínico hospitalar. Jornal de Pediatria, 80(5), 163-172. https://doi.org/10.1590/S0021-75572004000700008.

Oliveira, L. L. de, Gonçalves, A. C., Costa, J. S. D., \& Bonilha, A. L. L. (2016). Fatores maternos e neonatais relacionados à prematuridade. Revista da Escola de Enfermagem da USP, 50(3), 382-389. https://doi.org/10.1590/S0080-623420160000400002.

Rapoport, A.; \& Piccinini C. A. (2011). Maternidade e situações estressantes no primeiro ano de vida do bebê. Psico-USF, 16(2), 215-225. https://doi.org/10.1590/S1413-82712011000200010.

Ribeiro, C. C., Pachelli, M. R. O., Amaral, N. C. O., \& Lamônica, D. A. C. (2017). Habilidades do desenvolvimento de crianças prematuras de baixo peso e muito baixo peso. CoDAS, 29(1), 1-6. https://doi.org/10.1590/2317-1782/20162016058.

Ruble, L., McDuffie, A., King, A. S., \& Lorenz, D. (2008). Caregiver responsiveness and social interaction behaviors of young children with autism. Topics in Early Childhood Special Education, 28(3), 158-170, 2008. https://doi.org/10.1177/0271121408323009.

Silva, B. A. A., \& Braga, L. P. (2019). Fatores promotores do vínculo mãe-bebê no puerpério imediato hospitalar: uma revisão integrativa. Revista da SBPH, 22(1), 258-279.

Silva, J. L. P., Linhares, F. M. P., Barros, A. A., Souza, A. G., Alves, D. S., \& Andrade, P. O. N. (2018). Fatores associados ao aleitamento materno na primeira hora de vida em um hospital amigo da criança. Texto \& Contexto - Enfermagem, 27(4), 2-10. https://doi.org/10.1590/0104-07072018004190017. 
Research, Society and Development, v. 10, n. 16, e233101623286, 2021

(CC BY 4.0) | ISSN 2525-3409 | DOI: http://dx.doi.org/10.33448/rsd-v10i16.23286

Teixeira, G. A., Carvalho, J. B. L., Rocha, B. G., Pereira, S. A., \& Enders, B. C. (2018). Perfil de mães e o desfecho do nascimento prematuro ou a termo. Cogitare Enfermagem, 23(1). https://doi.org/10.5380/ce.v23i1.51409.

Zanettini, A., Urio, A., Souza, J. B., \& Geremia, D. S. (2019). As vivências da maternidade e a concepção da interação mãe-bebê: interfaces entre as mães primíparas adultas e adolescentes. Journal of Research: Fundamental Care Online, 11(3), 655-66. https://doi.org/10.9789/2175-531.2019.v11i3.655-663. 